\title{
Near infrared planar tumor imaging and quantification using nanosized Alexa 750-labeled phospholipid micelles
}

This article was published in the following Dove Press journal:

International Journal of Nanomedicine

18 May 2009

Number of times this article has been viewed

\author{
Aristarchos Papagiannaros \\ Amit Kale \\ Tatyana S Levchenko \\ Dmitry Mongayt \\ William C Hartner \\ Vladimir P Torchilin \\ Department of Pharmaceutical \\ Sciences and Center for \\ Pharmaceutical Biotechnology \\ and Nanomedicine, Northeastern \\ University, Boston, MA, USA
}

\begin{abstract}
A novel highly biocompatible near infrared nanosized contrast agent was developed and used for rapid tumor detection and quantification using planar optical imaging and analysis. With this in mind, the near infrared fluorescent dye Alexa 750 was covalently attached to polyethylene glycol-phosphatidylethanolamine (PEG-PE) conjugate, and double labeled (with Alexa and rhodamine) PEG-PE micelles were injected into mice and observed using planar optical imaging. Pixel intensity data from the tumor site were normalized versus the autofluorescence of the animal at the same time point and normalized as signal to noise over the scattered light from the various tissues of the mice. The detected signal from the tumor was higher than the background noise allowing for rapid detection of the tumor. The tumor was clearly visible within one hour. Some signal was also detected from the abdomen of the mice. As determined by microscopy analysis, other organs of accumulation were the liver and kidney, which corresponded well to the data from the whole body imaging animal studies.
\end{abstract}

Keywords: nanotechnology, nanomedicine, cancer imaging agents, near infrared imaging, micelles

\section{Introduction}

Near infrared (NIR) planar optical imaging is a powerful, simple, and easy to use method that has found increasing use for the observation of biological events. It uses the 'absorbance window' that exists in the near infrared part of the spectrum where light absorption by tissues is negligible. Therefore, light can penetrate up to a few centimeters through the body. ${ }^{1}$ Using NIR fluorescence and large stokes shift fluorophores that eliminate the back scattering of the excitation beam, whole body images of small animals can be taken with a better signal-to-noise ratio and greater clarity than in the visible spectrum, where light absorption allows only surface imaging. By using new highly stable fluorophores, images can be taken at exposure times of minutes and even real-time observations can take place, ${ }^{2}$ so that clear images are produced. For example, using this method it was possible to target and image various biological targets, such as estrogen growth factor receptors in breast cancer, ${ }^{3}$ vascular endothelial growth factor (VEGF) receptors in tumors and lymph nodes ${ }^{4}$ before surgery. Since NIR imaging does not require the complex instrumentation of other methods, such as positron emission tomography (PET) or magnetic resonance imaging (MRI), or the handling procedures associated with radioactive materials, it has gained attention for the visualization of tumors and cells in vivo, especially using nano-scaled materials. For instance, dendritic cells were labeled and monitored using NIR-emitting polymersomes, ${ }^{5}$ and endothelial cells were targeted and visualized using NIR-emitting CD31 antibody. ${ }^{6}$ 
Although NIR-imaging results in better images than in the visible spectrum, it presents some challenges. Thus, there is an intense scattering of the fluorescence by tissues, causing the distortion of the images and the loss of contrast, which is considered the main disadvantage of the NIR planar imaging. The quantification of the signal becomes challenging, since the scattered light interferes with the measurements. Increasing the signal intensity only worsens the intensity of light scattering. All approaches up to now have been focused mainly on the elimination of scattering by using various techniques either mathematically, such as in optical tomography, or physically, using multispectral imaging. ${ }^{7,8}$ Despite their elegance, these methods require complex instruments that make NIR-imaging a less practical choice, while the planar imaging is expected be useful in relatively simple everyday practice, allowing for the rapid identification of the internal structures of living subjects, in situ, and in real time. ${ }^{9}$ The approach is also expected to utilize organic dyes that can be safely administered to patients and avoids toxic metals such as cadmium or selenium used in quantum dots. ${ }^{10,11}$ However, both the appropriate contrast agent and the means for extraction of meaningful data from planar images still need to be better developed.

Various nanoparticulates have been developed specially for the NIR detection and imaging of tumors. ${ }^{12,13}$ In this context, polymeric micelles represent an established colloidal nanocarrier system that has great advantages for the delivery of various drugs and vaccines. Micelles self-assemble from various amphiphilic molecules upon their hydration and remain stable as long as their concentration is above the critical micellar concentration (CMC). Lipid-core micelles offer high biocompatibility when formed from polyethylene glycol-lipid conjugates. They have been used extensively for experimental anticancer therapy, especially with poorly water soluble drugs, such as paclitaxel or camptothecin, with photodynamic therapy, and as an imaging agent. ${ }^{14,15}$ Evidently, the micelles can be used as nanocarriers for NIR agents, and simultaneously NIR imaging, in turn, can be used for the visualization of such biodegradable nanocarriers in vivo, offering a direct method for observation of their fate (as well as of other different events) inside the body, which was previously impossible, ${ }^{16}$ both at the macroscopic and microscopic levels. ${ }^{16}$

Earlier efforts from our lab used radio-labeling to follow the biodistribution of the lipid-core micelles. However, the images produced with a gamma-camera were intrinsically limited by the low resolution of the gamma-camera and the contrast of the images. Tumors were optimally visible only after several hours, ${ }^{17}$ and significant signal intensity was often observed from various other internal organs. ${ }^{18-20}$

Here, we report the use of double-labeled micelles based on PEG-phosphatidylethanolamine (PEG-PE) conjugates, containing a NIR dye, Alexa 750, and rhodamine to passively target tumors in vivo and to visualize them with much higher detail and precision than previously observed using both whole body imaging and fluorescent microscopy. We also describe a rapid and simple methodology to evaluate the imaging capability of the contrast agent and quantify the signal in a comparable way between different time points and different animal subjects. The NIR images also provided an additional insight into the fate of such micelles in vivo.

\section{Experimental section Conjugation of Alexa $750 \mathrm{PE}$ to phospholipids}

Alexa succinimidyl ester (Invitrogen, Carlsbad, CA) was dissolved in chloroform and mixed with a $4 \mathrm{x}$ excess of DOPE- $\mathrm{NH}_{2}$ in the presence of tri-ethylamine, as recommended by the manufacturer. The mixture was kept overnight in darkness and the product purified by column chromatography (stationary phase silica gel, mobile phase chloroform-to-methanol 4:1 to 4:3). Purity of product was tested using thin layer chromatography (TLC; stationary phase silica gel, mobile phase chloroform-to-methanol 4:3). The conjugation was complete. Only Alexa conjugated to lipids was detected by the TLC at the end of the procedure ( $R f$ circa 0.6 ), while no unbound dye, co-eluted at the solvent front, was detected.

\section{Micelle preparation}

Alexa PE 750 micelles were prepared according to a protocol used routinely in our lab. Briefly, a chloroformic solution of PEG-PE 5000 was mixed with Alexa 750 PE (0.25\% molar) and rhodamine lysamine $\mathrm{PE}(0.25 \%$ molar $)$ in a round bottom flask ( 0.55 umole of phospolipids). Solvents were evaporated in a rotary evaporator and the lipid film was freeze dried overnight. Micelles were produced by hydration of the lipid film with phosphate-buffered saline (PBS) pH = 7.4 (0.2 ml) followed by bath sonication and a 30 min equilibration period. This standard protocol allows for the quantitative incorporation of lipid-modified moieties into the micelles formed. The size distribution of the micelles was measured using laser diffraction analysis (Counter N4; Beckman Coulter Inc., Fullerton, CA). The average hydrodynamic size of the micelles was $17.4 \pm 3.9 \mathrm{~nm}$. The micelles were stable in PBS, $\mathrm{pH}=7.4$ for a period of up to 15 days after their hydration. 


\section{Commercially available nanoparticles}

Kodak X-sight polymerized nanospheres, encapsulating an organic dye (excitation $761 \mathrm{~nm}$, emission 789 (Carestream Health, New Haven, CT) were used as is for comparison to the Alexa micelles.

\section{Tumor inoculation of mice}

Female Balb/c mice (Charles River Laboratories, Wilmington, MA) were used, following a protocol approved by Northeastern University Institutional Animal Care and Use Committee in accordance with 'Principles of Laboratory Animal Care' (NIH publication No. 85-23, revised in 1985). Animals had free access to food and water. Murine $4 \mathrm{~T} 1$ breast cancer cells were grown in Dulbecco modified Eagle's medium. 150,000 cells were suspended in $150 \mu \mathrm{l}$ of PBS and injected subcutaneously over the left flank. Tumors developed within a period of two weeks.

\section{Near infrared imaging of mice}

Contrast agents, prepared the same day, as described above, were injected via the tail vein at concentrations between 55 and $300 \mathrm{nmol} / \mathrm{kg}$, which is a somewhat smaller dose than used with other Alexa-labeled macromolecules (see for example $\mathrm{Li}$ Peng and colleagues ${ }^{21}$ ), however we have compensated the decreased dose with an increased exposure time. Mice were anesthetized (ketamine/xylazine, i.p.) and depilated (Nair, Princeton, NJ). Images were taken using a Kodak Image Station In Vivo FX (filters: excitation $710 \mathrm{~nm}$, emission $790 \mathrm{~nm}$ ) at different angles with an exposure time of $10 \mathrm{~min}$. At the end of the imaging, anesthetized mice were sacrificed, skin removed and images retaken. Images of the organs were also made, either inside the body or ex vivo. Samples were also taken for histological examination.

\section{Image analysis}

Images were analyzed using the Kodak Image Analysis Software or the NIH Image J software. Dorsal images of the animals were analyzed, so that the tumor directly faced the camera. Regions of interest (ROI) were determined for the tumor area, tumor background, that included tumor, liver and spleen sites and over the hip corresponding to the intrinsic fluorescence of the animal. The fluorescence mean intensities were extracted for each and the signal-to-noise ratio for the tumor area was calculated, using the pixel intensity values for the ROIs corresponding to the tumor and the local background, so that the fluorescence is presented as signal to noise instead of arbitrary pixel intensity values. In order to produce comparable data, the signal-to-noise ratio was normalized versus the ROI over the hip that represents the intrinsic fluorescence of the animal. This compensated for the difference in the overall luminosity of the pictures due to the differences of biodistribution of the contrast agent in the body at different time points.

\section{Histology}

Tissue samples from organs and tumor removed from animals immediately after their sacrifice were stored at $-80{ }^{\circ} \mathrm{C}$. Frozen samples were cryo-sectioned at a thickness of $6 \mu \mathrm{m}$ and observed under fluorescence microscopy (excitation $545 \mathrm{~nm}$ ). Slices were also stained by eosin and hematoxylin and observed with white field microscopy.

\section{Results and discussion}

Figure 1 presents a side view of animals injected with Alexalabeled micelles (A, B), so that the tumor is directly facing the camera. In this view, the tumor area shows higher fluorescence compared the area immediately surrounding it and to the rest of the body. At one hour, in these mice we observed the localized highly contrasted signal from the tumor area, and a similar quality image was obtained at two hours. We also observed an increasing signal from the abdomen of the animal that corresponded to the location of the bladder. The fluorescence from the bladder area, although lower at one hour compared to the tumor, increased with time. At two hours, it became the greater source of the fluorescence signal. The anatomical location of the sites emitting the fluorescence was verified by imaging of sacrificed animals after the skin was removed (data not shown). The quality of the images was improved by the choice of the viewing angle. The limited penetration of light into tissues during the optical imaging was useful, since different parts of the animal could be better visualized by reorienting the animal. Tissues that lay deeper inside the animal contribute only a diffuse light that is fairly uniform and can be eliminated (threshold) with great precision. This results in clear identification of features that are directly in front of the camera, while other phenomena were easily reduced or eliminated. This is not feasible using radio-labeled nanocarriers (micelles) since gammairradiation easily penetrates the tissues. For instance, using

${ }^{111}$ In-labeled micelles, the tumor site was visible only after significantly longer times and the images were noisy due to the high penetration of the gamma-irradiation. ${ }^{20}$

Figure 2 compares the ventral view of two animals, one (C) injected with Alexa-labeled micelles, and another (D) injected with the commercially available polymerized nanoparticles encapsulating a near infrared dye. The commercial 

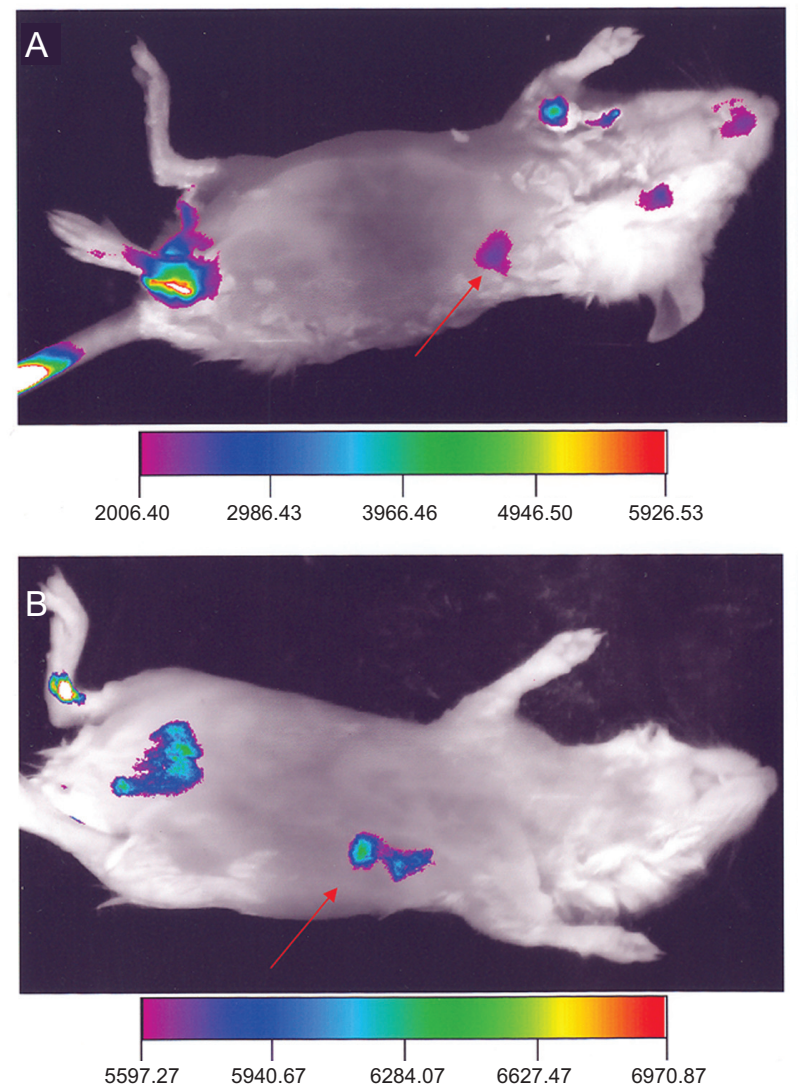
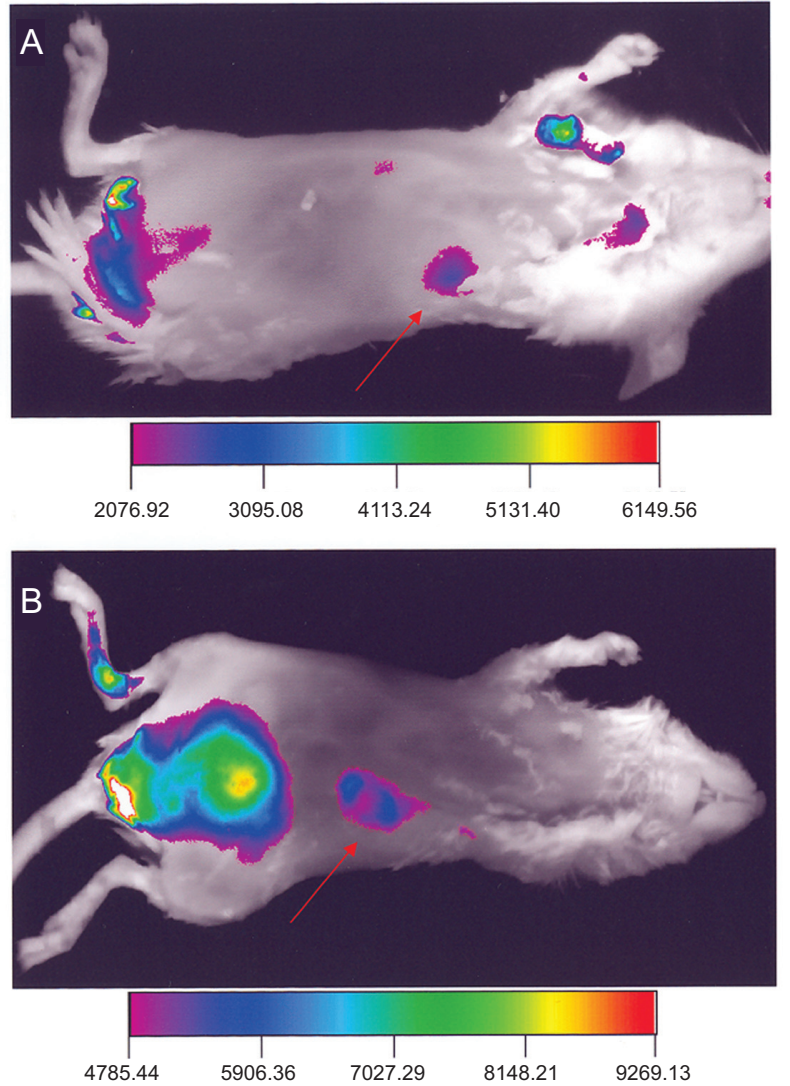

Figure I Side view of two animals (A and $\mathbf{B}$ ) injected with Alexa-labeled micelles, at one hour (left) and two hours (right) after the injection. Arrows point to the tumor.

nanoparticles exhibited a higher signal compared to the Alexa-micelles that was more uniformly distributed all over the body. The Alexa-labeled micelles exhibited a high signal mainly from the area over the bladder of the animal, while the commercial nanoparticles' localization corresponds to the liver. The fluorescence intensity observed in the abdomen (C) increased with time, doubling in value at between one and two hours. By contrast, the fluorescence from the liver of the animal D decreased slightly after two hours. The high signal in the abdomen, clearly observed in the frontal view is suggestive of a dissociation of the micelles, leading to the clearance of the near infrared dye. Gamma-camera studies with similar ${ }^{111}$ In-labeled micelles exhibited a very similar pattern. ${ }^{22}$ The pattern of the near infrared emission was also similar to studies of the stability of biodegradable nanoparticles using near infrared imaging. ${ }^{16}$ This conclusion is supported by the data from the Figure 3 (upper row), which shows a much higher deposition of rhodamine (the second label in our micelles) in the liver and kidney than in the tumor, suggesting the increased clearance of the micelle material through these organs. Both the results of the infrared imaging and of the whole body imaging suggest that the aggregate nature of the micelles contribute to this secretion pattern involving micelle dissociation. In Figure 3, lower row, hematoxylin and eosin staining of the slices verified the expected morphology of the tumor and organs that were free of metastasis. Figure 4 shows ex vivo images of one animal at the end of the experiment with the skin removed. The image reveals that at the end of the experiment, the main sources of fluorescence are the liver, the lower part of the gastrointestinal tract, and the bladder area.

Figure 5 shows the quantified background NIR signal from the area surrounding the tumor and from the control area over the hip using the planar whole body imaging of tumor-bearing mice during a four hour period. One can notice that neither the background noise from the tumor area nor the intrinsic fluorescence of the animal (the signal from the control area) remained constant during the imaging studies, although the changes in the signal are not dramatic or statistically significant in the case of the tumor area. The background noise, corresponding largely to the scattered light from internal organs (liver and spleen) as well as the tumor, initially slightly decreased after the one hour value of $1731 \pm 88$ A.U. As the biodistribution changed, the background in the organs and tumor slightly increased again at 

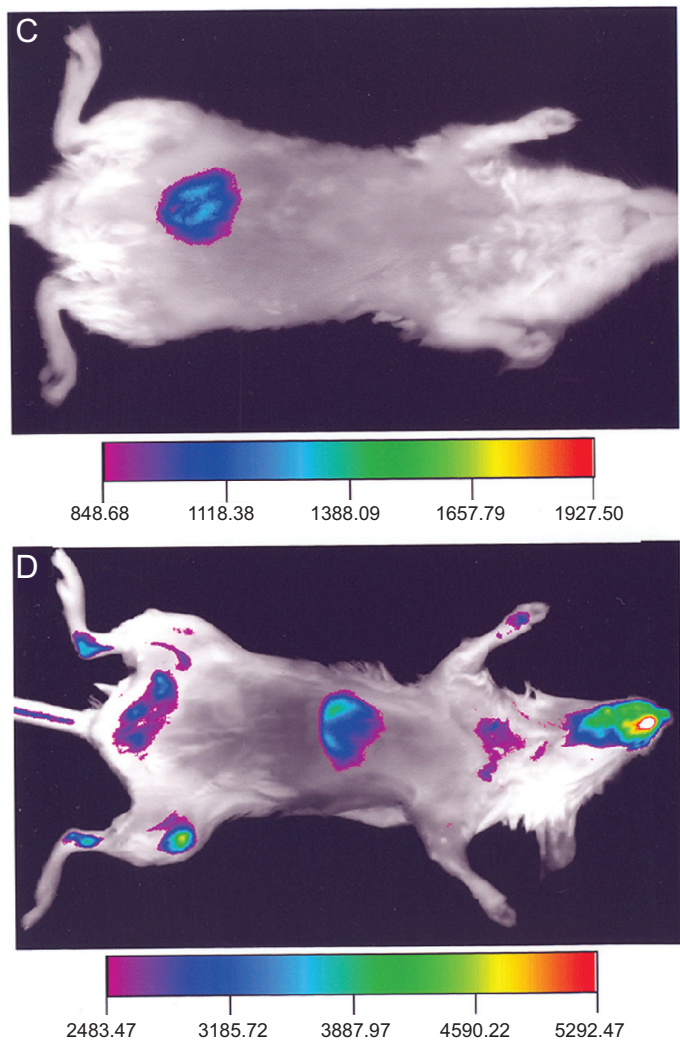

Figure 2 Ventral view of the animal injected with Alexa-labeled micelles (C) and another animal injected with Kodak X-Sight 76 I (D), at one hour (left) and two hours (right) after the injection.

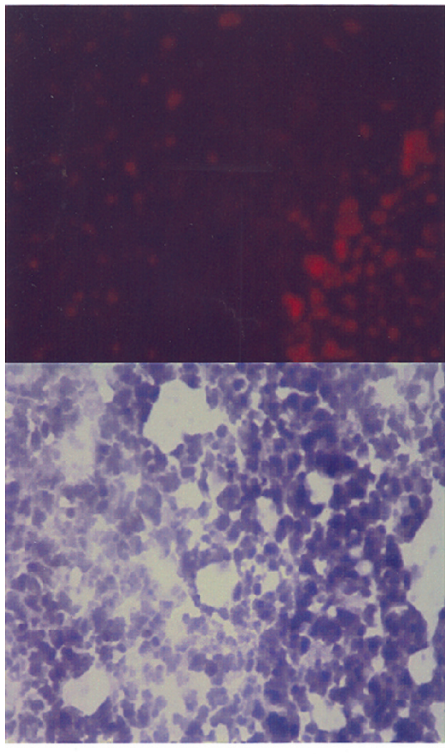

Tumor

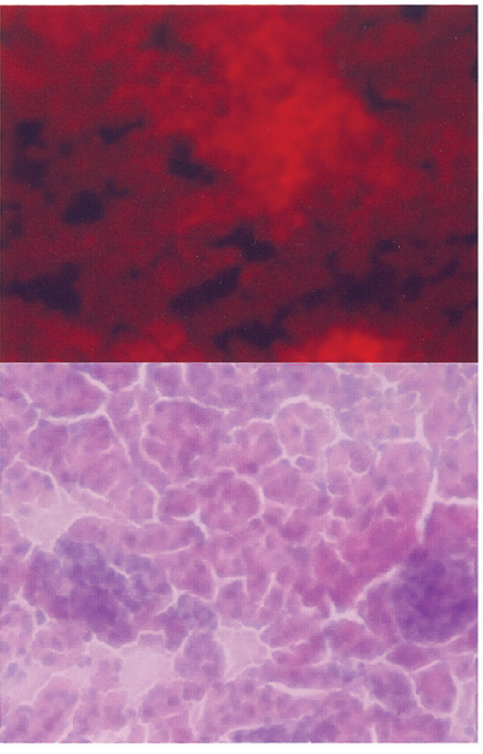

Kidney

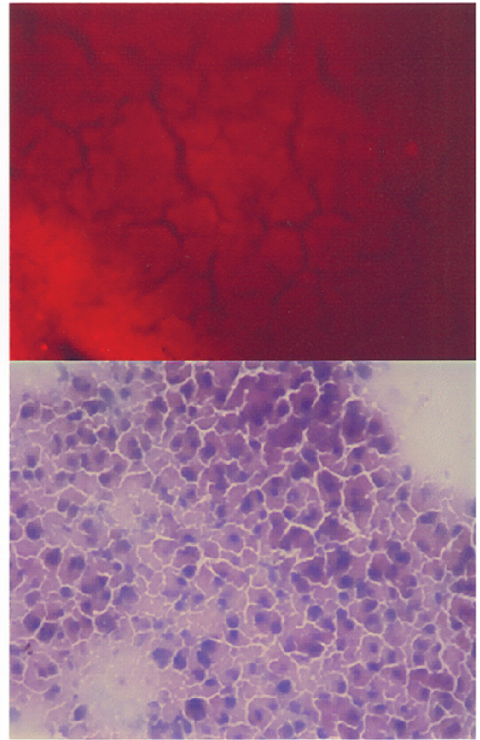

Liver

Figure 3 Cryosections of tumor, kidney, and liver removed from the animals injected with Alexa rhodamine-labeled micelles. Upper row: fluorescence microscopy imaging of rhodamine accumulation. Lower row: white field images of eosin and hematoxylin-stained sections. 

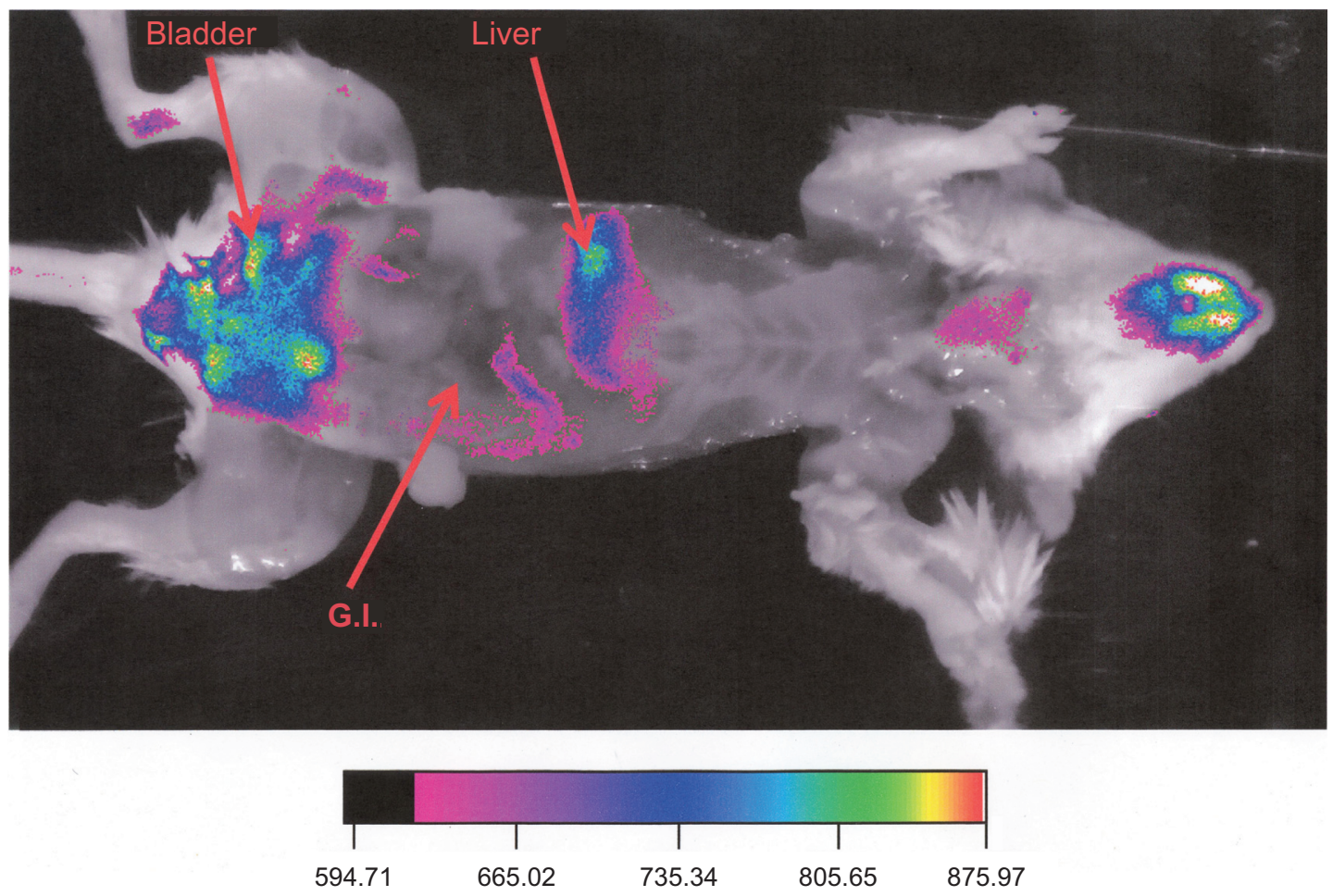

Figure 4 Ventral view of an animal injected with Alexa-labeled micelles at the end of the experiment with the skin removed.Tumor is visible in the side of the animal.

the end of the experiment to $1847 \pm 112$ A.U. On the other hand, the fluorescence signal from the animal body (control area) increased during the experiment, from $1542 \pm 77$ at one hour to $1900 \pm 105$ at four hours. The quantification of the signal in this case represents a major improvement, since it is based on normalized signal-to-noise data. This is a clear advantage over previous attempts with photons/gram of tissue using ex vivo data from organs excised from animals after the imaging. ${ }^{16}$ The inclusion of the whole body imaging and the generation of the data from all selected time points from the same animal decreased the variability of the measurements and augmented the physical relevance of the data. Whole body images provided an objective description of the imaging capacity of the set-up and the contrast agent and augmented the ability to make objective comparisons between different contrast agents. This was achieved because the two prime factors that introduce variability in the measurements were addressed: a) the difference in the background noise due to the time-dependent changes in the distribution of the agent in the body, and b) the difference in the overall luminosity of the image as the agent cleared from the animal. The normalization of the data is of critical importance for obtaining comparable results. ${ }^{23}$ In fact, multispectral imaging uses the data from different wavelengths that offer the same mechanism of normalization as the one employed here, since it compensates for the differences in the images during the course of imaging. ${ }^{24}$ The data obtained are still not as good as in case of the tomographic imaging. However, the main result here is the minimizing the effect of the background noise on the quality of the data. For instance, early work in the tomographic field concentrated on the effect of mathematical subtraction of noise, especially in heterogeneous media, ${ }^{25}$ or the normalization of the Born approximation ratio for the scattering of the fluorescence throughout the animal body. ${ }^{26}$ This approximation method successfully generated 3-D reconstructions of small animals ${ }^{27}$ and the quantification of the antitumor effect of treatment in vivo. ${ }^{28}$

Figure 6 shows that the signal-to-noise ratio from the Alexa-labeled micelles at the tumor remained higher than of areas near the tumor. It reached a value of $1.3 \pm 0.07$ within one hour and it remained at similar levels until the end of the experiment, reaching a value of $1.6 \pm 0.08$ at four hours. The signal-to-noise ratio was influenced by the different values obtained from the tissues surrounding the tumor ROI. The overall fluorescence of the animal remained high despite the fluctuations in the later values. We achieved the above mentioned high signal at the tumor site after only one hour of imaging, which allowed us to pinpoint the tumor site, so that its dimensions and boundaries were clearly defined. This signal was also higher than the surrounding tissues, such as 


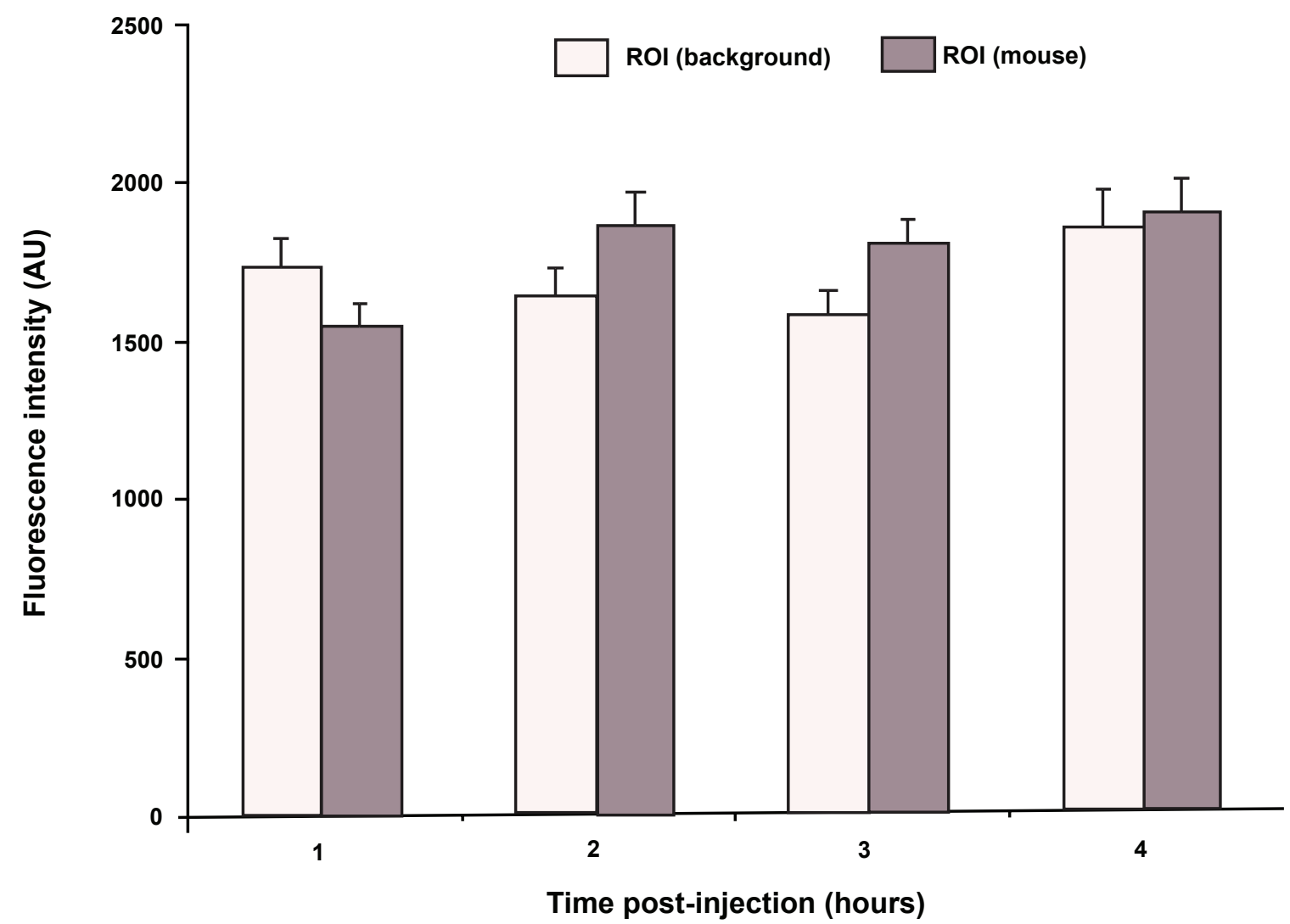

Figure 5 Fluorescence intensity of the background signal around the tumor and the intrinsic fluorescence of the animal body (control region of interest [ROI] over hip). The overall signal fluctuated with time as the biodistribution of the contrast agent changed.

Notes: $N=6$; bars represent standard error of the mean.

liver, where all nanoparticles tend to accumulate. This looks like a clear advantage of our version of the NIR optical imaging that allowed for the production of high quality images with a relatively simple and inexpensive instrument (in comparison to a gamma-camera or MRI system) and involved the use of environmentally friendly and less hazardous dyes. In addition, informative images can be obtained rather fast, which decreases the exposure of the subject to the contrast agent.

The imaging of polymeric near infrared dye-encapsulating commercial nanoparticles (Figure 2, lower row), showed a high localized signal in the liver but not in the bladder. Lipid nanoparticles, such as liposomes, have been used to monitor the extravasation of nanoparticles through the tumor vessels. ${ }^{29}$ Polymerosomes used for cell labeling remained intact and light-excitable inside the cells. ${ }^{5}$ Similar 'monolithic' nanoparticles can be much more appropriate for cell trafficking studies than nanoparticles made of inorganic materials such as quantum dots, due to their different toxicological profile.

To the best of our knowledge, our results present the first lipid-core micellar nanocarrier system using near infrared dyes for the whole body imaging. Recent research has emphasized the use of block copolymers to stabilize the easily degradable near infrared dye, indocyanine green. ${ }^{30}$ Low density lipoprotein used for breast tumor visualization in vivo using confocal microscopy ${ }^{31}$ was found to remain dispersed in the circulation, despite the presence of endogenous low-density lipoprotein. Finally, cross-linked micelle-like structures made of synthetic polymers allowed visualization of breast cancer xenographs, both in near infrared and using gamma-imaging. ${ }^{32}$

The major result of these experiments was the production of high quality images using organic dyes conjugated to nanosized micelles. They can produce high signal intensity that may be useful to visualize a variety of events, such as tumor uptake through an altered microcirculation. ${ }^{33}$ In case of quantum dots, despite their advantages in terms of optical quality, contrast and clarity, their use is hampered by serious toxicological implications, including the degradation of toxic metals within the cells. ${ }^{34-36}$ Previous work on near infrared imaging using labeled macromolecules had the disadvantage of low signal intensity and poor quality images. ${ }^{4,12}$ The conjugation of multiple molecules of near 


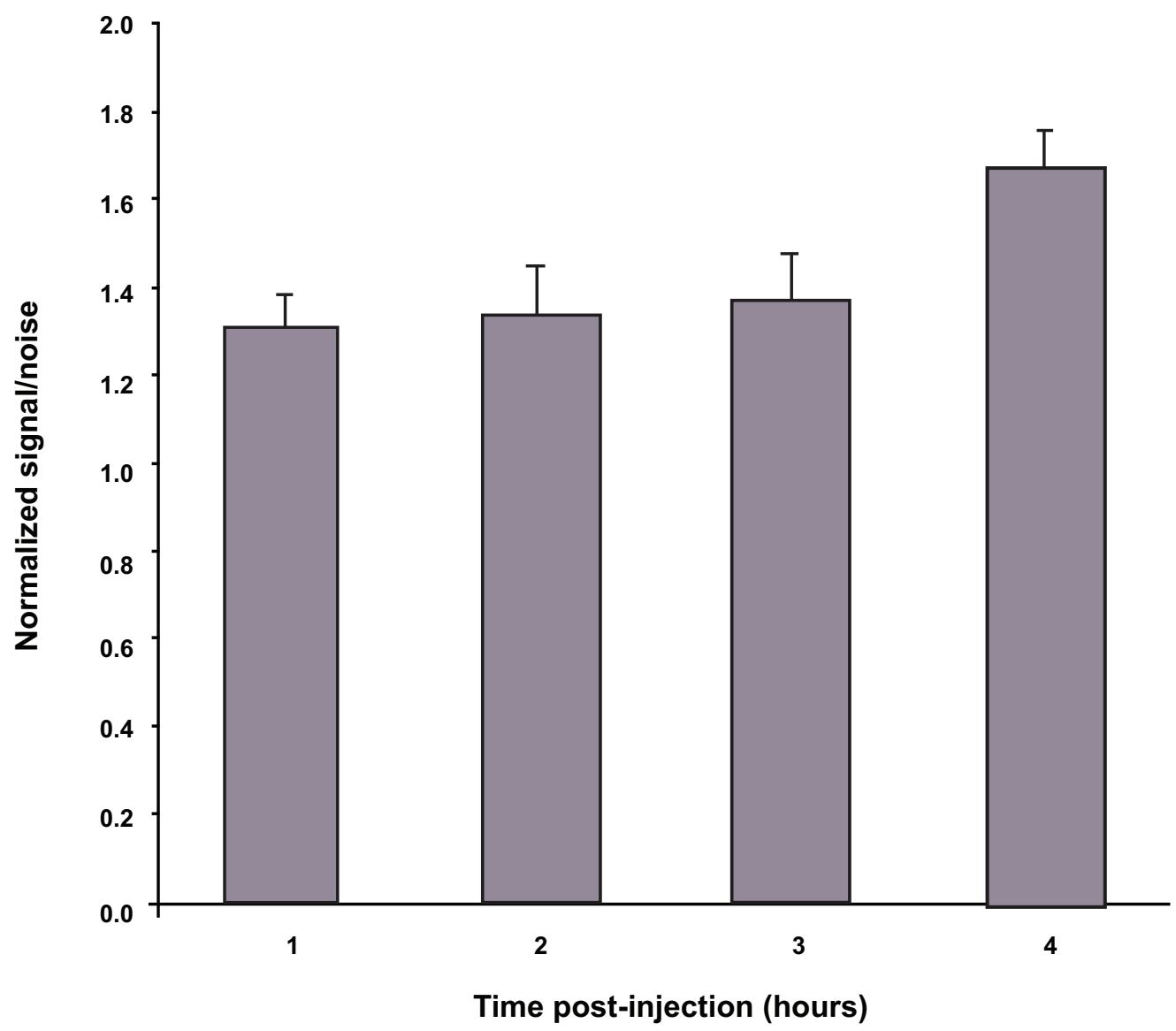

Figure 6 Normalized signal-to-noise ratio for the fluorescence of the tumor. Data show the ratio of the tumor fluorescence versus the background in the area around the tumor.

Notes: $\mathrm{N}=6$; bars represent standard error of the mean.

infrared dyes within micellar nanoparticles provides the means to increase the signal intensity and allows good signal quantification. In conclusion, this work demonstrates that the quantification of the accumulation of a contrast agent can be achieved using simple (and more relevant to everyday practice) instruments, and that highly biocompatible lipidcore PEG-PE-based micelles are convenient nanocarriers for NIR contrast agents.

\section{Acknowledgments}

This work was supported by NIH grant RO1 EB001961 to VP Torchilin. JR Upponi's excellent technical support is gratefully acknowledged. The authors report no conflicts of interest in this work.

\section{References}

1. Adams KE, Ke S, Kwon S, et al. Comparison of visible and near-infrared wavelength-excitable fluorescent dyes for molecular imaging of cancer. J Biomed Opt. 2007;12(2):024017.

2. Hassan M, Riley J, Chernomordik V, et al. Fluorescence lifetime imaging system for in vivo studies. Mol Imaging. 2007;6(4):229-236.
3. Ke S, Wen X, Gurfinkel M, et al. Near-infrared optical imaging of epidermal growth factor receptor in breast cancer xenografts. Cancer Res. 2003;63(22):7870-7875.

4. Backer MV, Levashova Z, Patel V, et al. Molecular imaging of VEGF receptors in angiogenic vasculature with single-chain VEGF-based probes. Nat Med. 2007;13(4):504-509.

5. Christian NA, Milone MC, Ranka SS, et al. Tat-functionalized nearinfrared emissive polymersomes for dendritic cell labeling. Bioconjug Chem. 2007;18(1):31-40.

6. Bogdanov AA Jr, Lin CP, Kang HW. Optical imaging of the adoptive transfer of human endothelial cells in mice using anti-human CD31 monoclonal antibody. Pharm Res. 2007;24(6):1186-1192.

7. Ntziachristos V, Culver JP, Rice BW. Small-animal optical imaging. J Biomed Opt. 2008;13(1):011001.

8. Themelis G, Yoo JS, Ntziachristos V. Multispectral imaging using multiple-bandpass filters. Opt Lett. 2008;33(9):1023-1025.

9. Pierce MC, Javier DJ, Richards-Kortum R. Optical contrast agents and imaging systems for detection and diagnosis of cancer. Int J Cancer. 2008;123(9):1979-1990.

10. Choi AO, Cho SJ, Desbarats J, Lovric J, Maysinger D. Quantum dotinduced cell death involves Fas upregulation and lipid peroxidation in human neuroblastoma cells. J Nanobiotechnology. 2007;5:1.

11. Clarke S, Nadeau J, Bahcheli D, Zhang Z, Hollmann C. Quantum dots as phototoxic drugs and sensors of specific metabolic processes in living cells. Conf Proc IEEE Eng Med Biol Soc. 2005;1:504-507.

12. Bloch S, Lesage F, McIntosh L, Gandjbakhche A, Liang K, Achilefu S. Whole-body fluorescence lifetime imaging of a tumor-targeted nearinfrared molecular probe in mice. J Biomed Opt. 2005;10(5):054003. 
13. le Masne de Chermont Q, Chaneac C, Seguin J, et al. Nanoprobes with near-infrared persistent luminescence for in vivo imaging. Proc Natl Acad Sci US A. 2007;104(22):9266-9271.

14. Torchilin VP. Targeted pharmaceutical nanocarriers for cancer therapy and imaging. Aaps J. 2007;9(2):E128-E147.

15. Torchilin VP. Micellar nanocarriers: pharmaceutical perspectives. Pharm Res. 2007;24(1):1-16.

16. Melancon MP, Wang W, Wang Y, et al. A novel method for imaging in vivo degradation of poly(L-glutamic acid), a biodegradable drug carrier. Pharm Res. 2007;24(6):1217-1224.

17. Elbayoumi TA, Torchilin VP. Enhanced accumulation of long-circulating liposomes modified with the nucleosome-specific monoclonal antibody 2C5 in various tumours in mice: gamma-imaging studies. Eur J Nucl Med Mol Imaging. 2006;33(10):1196-1205.

18. Elbayoumi TA, Pabba S, Roby A, Torchilin VP. Antinucleosome antibodymodified liposomes and lipid-core micelles for tumor-targeted delivery of therapeutic and diagnostic agents. J Liposome Res. 2007;17(1):1-14.

19. Lukyanov AN, Hartner WC, Torchilin VP. Increased accumulation of PEG-PE micelles in the area of experimental myocardial infarction in rabbits. J Control Release. 2004;94(1):187-193.

20. Roby A, Erdogan S, Torchilin VP. Enhanced in vivo antitumor efficacy of poorly soluble PDT agent, meso-tetraphenylporphine, in PEG-PE-based tumor-targeted immunomicelles. Cancer Biol Ther. 25 2007;6(7):1136-1142.

21. Peng L, Liu R, Marik J, Wang X, Takada Y, Lam KS. Combinatorial chemistry identifies high-affinity peptidomimetics against alpha4beta1 integrin for in vivo tumor imaging. Nat Chem Biol. 2006;2(7):381-389.

22. Roby A, Erdogan S, Torchilin VP. Solubilization of poorly soluble PDT agent, meso-tetraphenylporphin, in plain or immunotargeted PEG-PE micelles results in dramatically improved cancer cell killing in vitro. Eur J Pharm Biopharm. 2006;62(3):235-240.

23. Ntziachristos V, Turner G, Dunham J, et al. Planar fluorescence imaging using normalized data. J Biomed Opt. 2005;10(6):064007.

24. Vogel A, Chernomordik VV, Riley JD, et al. Using noninvasive multispectral imaging to quantitatively assess tissue vasculature. $J$ Biomed Opt. 2007;12(5):051604.

25. Gao M, Lewis G, Turner GM, Soubret A, Ntziachristos V. Effects of background fluorescence in fluorescence molecular tomography. Appl Opt. 2005;44(26):5468-5474.
26. Soubret A, Ripoll J, Ntziachristos V. Accuracy of fluorescent tomography in the presence of heterogeneities: study of the normalized Born ratio. IEEE Trans Med Imaging. 2005;24(10):1377-1386.

27. Soubret A, Ntziachristos V. Fluorescence molecular tomography in the presence of background fluorescence. Phys Med Biol. 2006;51(16) 3983-4001.

28. Choi HK, Yessayan D, Choi HJ, et al. Quantitative analysis of chemotherapeutic effects in tumors using in vivo staining and correlative histology. Cell Oncol. 2005;27(3):183-190.

29. Liu P, Zhang A, Zhou M, Xu Y, Xu LX. Real time 3D detection of nanoparticle liposomes extravasation using laser confocal microscopy. Conf Proc IEEE Eng Med Biol Soc. 2004;4:2662-2665.

30. Rodriguez VB, Henry SM, Hoffman AS, Stayton PS, Li X, Pun $\mathrm{SH}$. Encapsulation and stabilization of indocyanine green within poly(styrene-alt-maleic anhydride) block-poly(styrene) micelles for near-infrared imaging. J Biomed Opt. 2008;13(1):014025.

31. Song L, Li H, Sunar U, et al. Naphthalocyanine-reconstituted LDL nanoparticles for in vivo cancer imaging and treatment. Int JNanomedicine. 2007;2(4):767-774.

32. Yang Z, Zheng S, Harrison WJ, et al. Long-circulating near-infrared fluorescence core-cross-linked polymeric micelles: synthesis, characterization, and dual nuclear/optical imaging. Biomacromolecules. 2007;8(11):3422-3428.

33. Voura EB, Jaiswal JK, Mattoussi H, Simon SM. Tracking metastatic tumor cell extravasation with quantum dot nanocrystals and fluorescence emission-scanning microscopy. Nat Med. 2004;10(9):993-998.

34. Hardman R. A toxicologic review of quantum dots: toxicity depends on physicochemical and environmental factors. Environ Health Perspect. 2006;114(2):165-172.

35. Mancini MC, Kairdolf BA, Smith AM, Nie S. Oxidative quenching and degradation of polymer-encapsulated quantum dots: new insights into the long-term fate and toxicity of nanocrystals in vivo. $\mathrm{J} \mathrm{Am} \mathrm{Chem}$ Soc. 2008;130(33):10836-10837.

36. Shiohara A, Hoshino A, Hanaki K, Suzuki K, Yamamoto K. On the cyto-toxicity caused by quantum dots. Microbiol Immunol. 2004;48(9): 669-675.
International Journal of Nanomedicine

\section{Publish your work in this journal}

The International Journal of Nanomedicine is an international, peerreviewed journal focusing on the application of nanotechnology in diagnostics, therapeutics, and drug delivery systems throughout the biomedical field. This journal is indexed on PubMed Central,

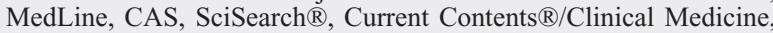

\section{Dovepress}

Journal Citation Reports/Science Edition, EMBase, Scopus and the Elsevier Bibliographic databases. The manuscript management system is completely online and includes a very quick and fair peer-review system, which is all easy to use. Visit http://www.dovepress.com/ testimonials.php to read real quotes from published authors. 